\title{
Nonmagnetic Rigid and Flexible Outer Sheath with Pneumatic Interlocking Mechanism for Minimally Invasive Surgical Approach
}

\author{
Hiromasa Yamashita ${ }^{1}$, Siyang Zuo ${ }^{1}$, Ken Masamune ${ }^{1}$, Hongen Liao ${ }^{2}$, \\ and Takeyoshi Dohi ${ }^{1}$ \\ ${ }^{1}$ Graduate School of Information Science and Technology, The University of Tokyo, Japan \\ ${ }^{2}$ Graduate School of Engineering, The University of Tokyo, Japan
}

\begin{abstract}
We developed a nonmagnetic rigid and flexible outer sheath with pneumatic interlocking mechanism using flexible toothed links and a wiredriven bending distal end. The outer sheath can be switched between rigid and flexible modes easily depending on surgical scenes, and the angle of its distal end can be controlled by three nylon wires. All components of flexible parts are made of MRI-compatible nonmagnetic plastics. We manufactured the device with 300-mm long, 16- $\mathrm{mm}$ outer diameter, 7-mm inner diameter and 90-mm bending distal end. Holding power of the device in rigid mode was maximum $3.6 \mathrm{~N}$, which was sufficient for surgical tasks in body cavity. In vivo experiment using a swine, our device performed smooth insertion of a flexible endoscope and a biopsy forceps into reverse side of the liver, intestines and spleen with a curved path. In conclusion, our device shows availability of secure approach of surgical instruments into deep cavity.
\end{abstract}

\section{Introduction}

Minimally invasive surgery (MIS) is in favor of closed or local surgery with fewer traumas unlike conventional invasive open surgery. In MIS, endoscopic devices and instruments are usually used to enable remote control surgical procedures with indirect observation of the surgical field through patients' body surface such as abdominal wall and chest wall into their body cavity, or through natural orifice such as mouth, urethra and anus. MIS allows patients to have shorter hospitalization time, outpatient treatment and earlier rehabilitation into society.

Various endoscopic devices and instruments have been researched and developed to improve surgical dexterity, and to reduce specific risks and difficulties in endoscopic surgery. For example a dexterous robotic manipulator added multiple degrees of freedom (DOFs) to operation of the instruments and thus it improved surgical performance [1-3]. So-called master-slave robotic manipulators, such as previously-commercialized da Vinci ${ }^{\circledR}$ Surgical System, enable surgeons to work with precise operation [4]. However, some problems remain unsolved in these surgical robotic systems. First, familiar laparoscopic surgery, which is one of the endoscopic surgeries, requires a sufficient space below the abdominal wall for surgical procedures. A pneumoperitoneum is commonly used to secure the space, but some 
complications have been reported from this method such as compression of organs by filled gas and solve out of $\mathrm{CO}_{2}$ gas into vessels. Second, laparoscopic surgery is useful when the affected area can be approached from the anterior of the body, however, it is difficult to approach the target in a deep and narrow area or reverse side of organs. Some research groups have developed flexible manipulators with a large curved radius. Ikuta et al. developed a micromanipulator to approach inaccessible regions [5]. Other flexible manipulators have been developed using shape memory alloys [6] or a wire-driven mechanism [7]. These manipulators can be inserted in the narrow space and approach affected area, however stabilized path and local surgical space can not be secured for other instruments such as a laparoscope, a forceps etc.

To solve this issue, some systems with lockable sheaths or hybrid locking and relaxing function have been developed. Robert et al. applied this idea to medical applications [8]. Snake shaped robot, called HARP, was also developed [9]. Yagi et al. developed a guiding device including an inner channel, which uses flexible manipulators to approach deep regions [10]. These manipulators have some limitations because their mechanisms of distal bending part are very complicated and their diameters must be larger than the size required for laparoscopic surgery. Moreover, it is difficult to make their components nonmagnetic material to realize MRI-compatibility. MRIguided surgery is effective for such as minimally invasive therapy of tumors throughout the body [11].

On inserting an instrument into the area of interest without any damages to around tissues, surgical instruments require special function to switch a flexible mode for free insertion and a rigid mode for stabilizing the outer sheath. In this study, we developed a rigid and flexible outer sheath with pneumatic locking mechanism using flexible toothed links and with wire-driven active-bending distal end for laparoscopic surgery.

This paper reports on (1) the mechanism switching between a flexible and a rigid modes, (2) a prototype of the outer sheath device with an active-bending distal end and (3) mechanical performance evaluation of the device. Moreover, we evaluated it (4) in vivo experiment using a swine.

\section{Materials and Methods}

\subsection{Outer Sheath Design}

The outer sheath provides a secure approach path for the other surgical instruments inside the human body. The sheath can be flexible and twisted into any shape passively and can still retain its shape against external forces or insertion frictional force from inserted instruments. Before inserting flexible surgical instruments, the surgeon inserts the outer sheath with flexible mode manually through the narrow gap between the safety areas under endoscopic or MRI-guidance. When insertion direction of the outer sheath should be controlled, its wire-driven distal end is bent selectively. After the sheath approaches the target, the surgeon locks the shape and then inserts flexible instruments easily through the internal path made by the sheath. 

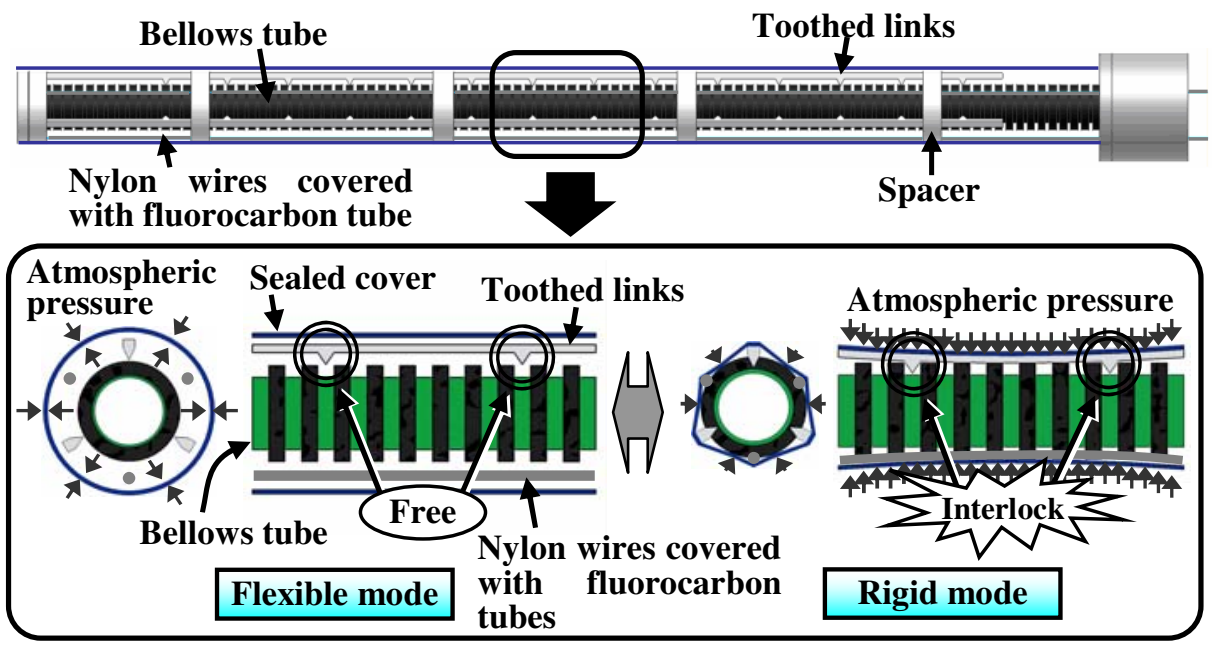

Fig. 1. Switching mechanism of the outer sheath between a flexible and a rigid modes. When there is no vacuum, the toothed links and the bellows tube can take any shape in a sealed clearance space. On the other hand when the pneumatic pump creates a vacuum, the links are pressed and meshed with the ditch of the tube, locking the shape of the sheath.

The outer sheath can be switched between a flexible and a rigid modes easily (Fig. 1). This mechanism consists of three flexible toothed links, a centered bellows tube and a surrounding sealed cover. In the flexible mode, inside air pressure between the bellows tube and the sealed cover is equal to atmospheric pressure. Because there becomes a sealed clearance space between the bellows tube and the sealed cover, teeth of the toothed links disengage from chased external surface of the bellows tube. In the rigid mode, sealed clearance space between the bellows tube and the sealed cover is evacuated by discharging the internal air, and the atmospheric pressure presses the toothed links into the external surface of bellows tube. These interlocked links and bellows tube lock the shape of the outer sheath. And three nylon wires are passing through the clearance space along the bellows tube to bend the distal end of the outer sheath. These wires are covered with unshrinkable fluorocarbon tubes.

\subsection{System Configuration}

System configuration of the prototype of the outer sheath is shown in Fig. 2. The outer sheath has an outer diameter of $16 \mathrm{~mm}$, inner diameter of $7 \mathrm{~mm}$, length of 300 $\mathrm{mm}$ and can achieve a minimum curvature radius of $45 \mathrm{~mm}$. The outer sheath, as described above, consists of three flexible toothed links, a bellows tube, three nylon wires covered with fluorocarbon tubes and a polyethylene sealed cover. The toothed links and bellows tube are flexible, and their shapes are free and changeable. The three toothed links are placed 120 degrees apart, making it possible to lock the outer sheath in any direction in three-dimensional space. The ticks of the bellows tube are 2 $\mathrm{mm}$ apart, and the locking teeth on the links are $17 \mathrm{~mm}$ apart. All parts of the outer sheath are made of plastic, ensuring MRI-compatibility. The bellows tube is connected to a vacuum pump (DTC-41, ULVAC KIKO INC., Japan) and switching controller (V030E1, Koganei, Japan) to change condition of the outer sheath between 


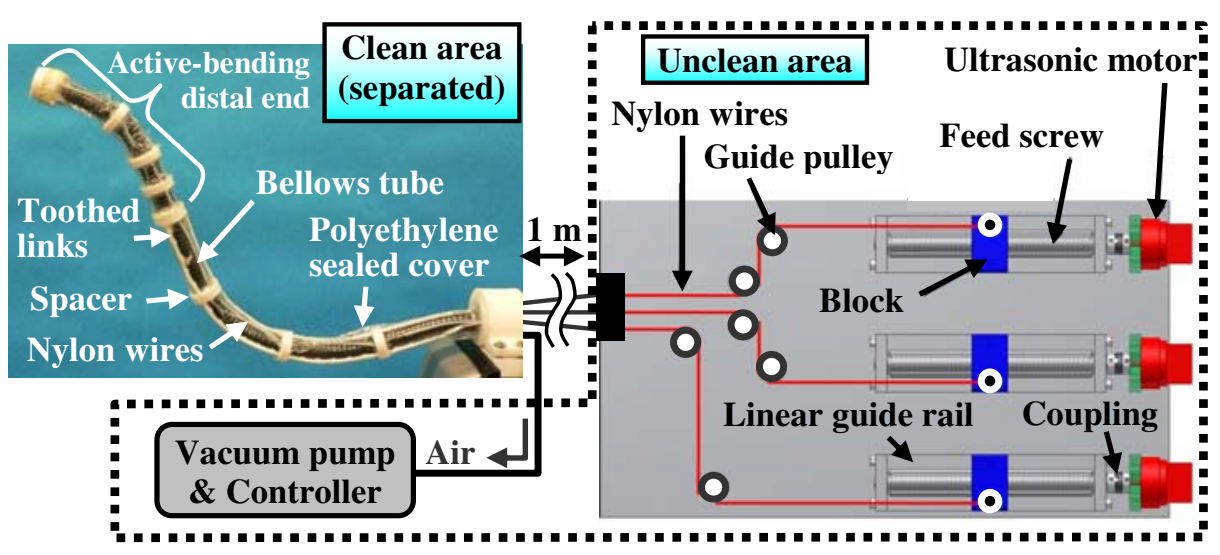

Fig. 2. System configuration of the outer sheath device. Rigid and flexible modes can be switched by the vacuum pump and controller. Bending angle of the distal end is controlled by three sets of ultrasonic motors. The outer sheath is $1-\mathrm{m}$ separated from other parts for cleanness.

a flexible and a rigid modes. In the rigid mode, flexible toothed links engage the bellows tube and they are locked by the vacuum. The shape of the outer sheath can be locked even with a twisted shape. When the vacuum is released, the sheath switches back to the flexible mode. Using this mechanism, it is possible to maintain a rigid shape for the outer sheath whenever a vacuum is continuous. In addition the device has a $90-\mathrm{mm}$ wire-driven distal end. The three nylon wires, which are also placed 120 degrees apart, drive bending angle of this part to arbitrary direction by ultrasound motors (USR30E3N, Shinsei Corporation, Japan) with couplings and feed screws. Surgeons can control bending angle by rotational three knobs. Materials of the linear-guides are also plastic to realize MRI-compatibility. The outer sheath is specifically separated from the actuators' part and the vacuum pump to be cleanable and sterilizable for clinical use.

\subsection{Wire-Driven Bending Mechanism}

Each nylon wire is passed through spacers aligned around the bellows tube. When the wire is pulled, length of the wire at one unit between adjacent two spacers is $l_{i}$ changes to $l_{i}$ ' depending on the bending angle $\theta_{i}$ (Fig. 3(A)). Relationship between $l_{i}$ and $l_{i}$ ' are described by an equation (1) with geometric relations in Fig. 3(A).

$$
\frac{l_{i}}{2}: b \frac{\theta_{i}}{2}=b \sin \frac{\theta_{i}}{2}+\frac{l_{i}^{\prime}}{2}: b \sin \frac{\theta_{i}}{2}
$$

Displacement of the wire $\Delta l_{i}$ is, when $\theta_{i}$ is not zero, described by an equation (2) and

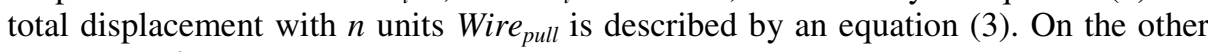
hand when $\theta_{i}$ is zero, $\Delta l_{i}$ and Wire $_{\text {pull }}$ are also zero.

$$
\Delta l_{i}=l_{i}-l_{i}{ }^{\prime}=l_{i}-\left(\frac{2 l_{i}}{\theta_{i}}-2 b\right) \sin \frac{\theta_{i}}{2}(2), \quad \text { Wire }{ }_{\text {pull }}=\sum_{i=1}^{n} \Delta l_{i}
$$

Relationship between total bending angle and total wire displacement is shown in Fig. 3(B) with 8 units including 4-units distal end of the prototype shown in Fig. 2. Each bending range of one unit is about 22.5 degrees and in case of bending with only the distal end part, maximum bending angle is about 90 degrees. On the other hand bending of the whole device with eight units achieves about 180 degrees. 

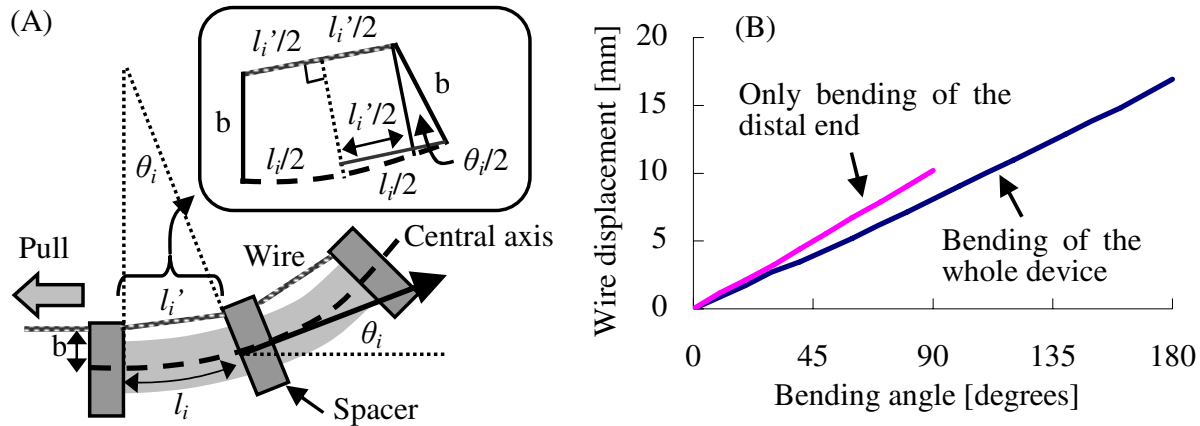

Fig. 3. (A) Wire-driven bending model at one unit between adjacent two spacers. Pulling wire performs each unit's bending motion. (B) Relationship between bending angle and wire displacement of only with the distal end and the whole device.

(A) Flexible mode

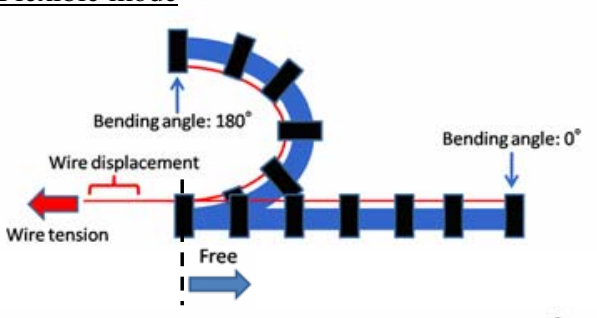

(B) Rigid mode

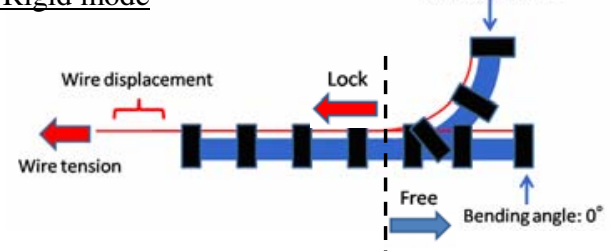

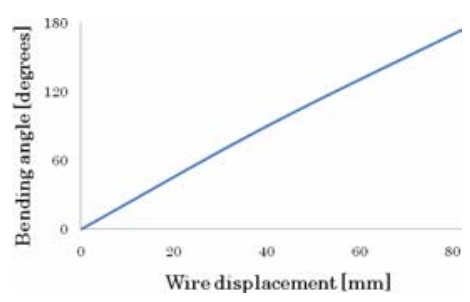

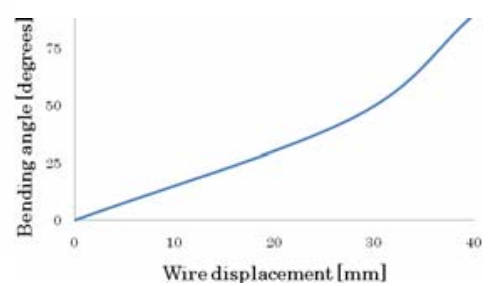

Fig. 4. Active-bending characteristics of the outer sheath by pulling one wire. (A) Result in flexible mode. (B) Result in rigid mode.

\section{Results}

\subsection{Mechanical Performance Evaluations}

First, we examined bending characteristics by pulling wire in the flexible and the rigid modes. In the flexible mode, only one wire was used to bend whole outer sheath including the distal end. In the rigid mode, the active-bending distal end was only bent. In the flexible mode, bending angle of 180 degrees was achieved with $85-\mathrm{mm}$ wire displacement and 11.7-N wire tension (Fig. 4(A)). In the rigid mode, bending angle of 90 degrees was achieved with 40-mm wire displacement and $16.6-\mathrm{N}$ wire tension (Fig. 4(B)). Second, we examined limited holding powers of outer sheath as keeping its shape using only the toothed links interlocking mechanism in rigid modes. Limited 

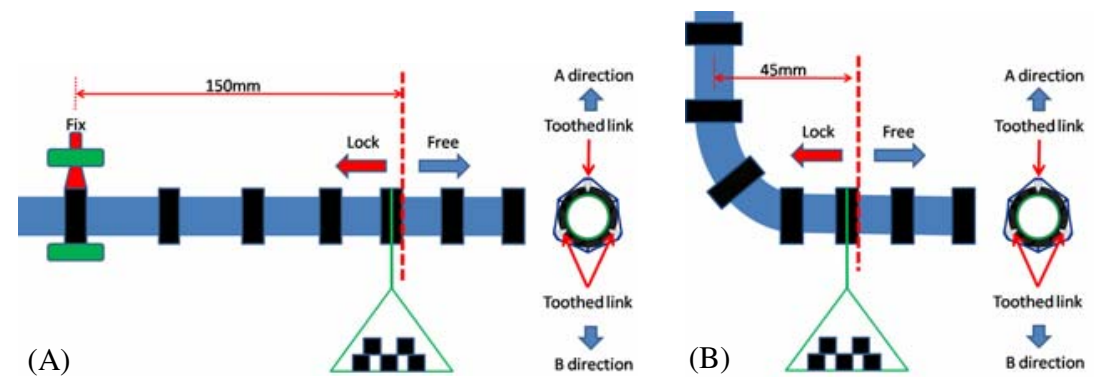

Fig. 5. Measurement of holding power in rigid mode. (A) Load on direction B from direction A in straight condition $\left(0^{\circ}\right)$. (B) Load on direction B from direction A in curved condition $\left(90^{\circ}\right)$.

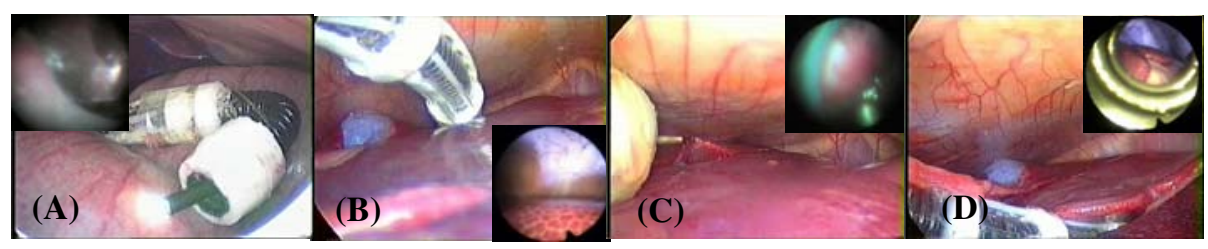

Fig. 6. Laparoscopic broad views with fiberscope local views using our device inserted into abdominal cavity of swine. (A) Colon observation with a widely curved distal end inserting a fiberscope, which shows reverse side of the colon. (B) The outer sheath goes into the gap between liver and abdominal membrane. Fiberscope shows space between liver and abdominal membrane. (C) Liver lobes clamping image by a biopsy forceps inserted the outer sheath. Fiberscope shows clamped liver lobes. (D) The outer sheath goes into the gap between spleen, liver and stomach. Fiberscope shows between their organs.

holding powers were values where the loaded outer sheath's shape started to deform, that is, the toothed links started to be unlocked, and we could observe it by the eye. The outer sheath was fixed at the second spacer from based side and measured in two cases, one was in straight condition $\left(0^{\circ}\right)$ (Fig. $5(\mathrm{~A})$ ), and the other was in curved condition $\left(90^{\circ}\right)$ (Fig. 5(B)). Loaded direction was downward vertically in both cases. Maximum load without deformation of the shape were $1.6 \mathrm{~N}$ in case (A) and $3.6 \mathrm{~N}$ in case (B).

\subsection{In vivo Experiment}

In in vivo experiment, we tested usability of the device in the abdominal cavity of a swine (male, $45.5 \mathrm{~kg}$ ). Inserted instruments into the outer sheath were a $2.8-\mathrm{mm}$ fiberscope (SUMITA Optical glass, Inc., Japan), a 6-mm high resolution fiberscope (VHF61, KEYENCE CORPORATION, Japan) and a 1.75-mm biopsy forceps (BF1812SF, FUJINON TOSHIBA ES SYSTEMS CO. LTD, Japan). A 2.8-mm fiberscope and a $1.75-\mathrm{mm}$ biopsy forceps could be inserted at the same time. We used a normal laparoscope to observe and confirm position and shape of the device.

First, we tried observation of the colon from the back side with a 2.8 -mm fiberscope (Fig. 6(A)). Second, we inserted the device into the gap between liver and abdominal membrane to observe a back side of the liver with a 6-mm fiberscope (Fig. 6(B)). Third, we clamped liver lobes surface by a biopsy forceps with a $2.8-\mathrm{mm}$ 
fiberscope view (Fig. 6(C)). Fourth, we inserted the device into the gap between spleen, liver and stomach. These tasks were not supported by any forceps (Fig. 6(D)).

\section{Discussion}

In mechanical performance evaluations, we confirmed that our device performed large bending angle in both flexible and rigid modes, which enabled switching between 0 \pm 180 -degrees bending of the whole device in the flexible mode and $0 \sim \pm 90$-degrees bending of the distal end of the device in the rigid mode depending on various surgical cases. In this study only a $90-\mathrm{mm}$ distal end could be actively bent in the rigid mode, if the length of this part is variable, our device may enable more dexterous and secure approach to apply to complicated deep cavity. And if the number of active DOF increases, the wire-driven mechanism would become more complex, however its appearance is not so changed because the wires are thin to the outer sheath. More in vivo experiments should be needed to evaluate the suitable constitution of active-bending parts and passive flexible parts for various surgical applications. About holding powers of outer sheath to keep its shape in the rigid mode, it is said that $5-\mathrm{N}$ is required for forceps power in general laparoscopic surgery to raise and hold heavy organs [12]. However our device purposes only insertion of flexible instruments into gap between organs, therefore even 1.58 $\mathrm{N}$ in straight shape and $3.58 \mathrm{~N}$ in 90-degrees curved shape may be sufficient against external pressure from organs and internal pressure from inserted instruments through the bellows tube, and this validity was proved in in vivo experiment.

Result of in vivo experiment indicated usability of our device in practical clinical condition. The outer sheath could go into gaps between organs, abdominal wall and membranes in deep cavity with wire-driven bending motion of the distal end as switching the flexible and the rigid modes, and flexible instruments such as fiberscope and biopsy forceps could be passed through the bellows tube into target deep space. On the other hand in manipulation of biopsy forceps, low resolution of the $2.8-\mathrm{mm}$ fiberscope was not enough to observe local operative fields. And some methods of real-time secure detection of position and shape of the device were thought to be needed besides a normal laparoscope. In the outer sheath, some small magnetic positioning sensors are mountable to track approach path of the device. Or more specifically, MRI-guided surgery is possible due to MRI-compatibility of the device.

Moreover our device is useful for natural orifice transluminal endoscopic surgery (NOTES) with improvements to downsize the outer diameter under $10 \mathrm{~mm}$ and to dilate inner channel. In NOTES, transluminal approach path is generally twisted up in 3D space and it is significant to transport required flexible instruments to deep operative field in safely. Our device can provide an arbitrary secure path for various flexible instruments by itself, and size of inner channel diameter, total length and length of activebending distal end are customized freely for on demand minimally invasive surgery.

\section{Conclusion}

In order to secure an approaching path for minimally invasive surgical instruments especially used in deep operative fields, we developed a nonmagnetic rigid and flexible outer sheath with pneumatic interlocking mechanism using flexible toothed links and an active-bending wire-driven distal end. We evaluated mechanical performances of bending angle and holding power of the device to use in practical clinical condition. In vivo 
experiment using a swine indicated validity of large bending angle up to \pm 180 degrees and sufficient holding power up to $3.6 \mathrm{~N}$ in the flexible and the rigid modes, and that some flexible instruments can be inserted into deep areas in abdominal cavity. These results show sufficient possibility of use of the device in NOTES with some improvements in tracking method for minimally invasive image-guided surgery.

Acknowledgment. We wish to thank Dr. Hashizume, Dr. Tomikawa, Dr. Tanoue, Dr. Ieiri and Dr. Konishi in Kyushu University for cooperation in vivo experiment. This work was supported in part by Grant-in-aid for Scientific Research (18680042, 1710008) of Ministry of Education, Culture, Sports, Science and Technology (MEXT) in Japan, the Special Coordination Funds for Promoting Science and Technology commissioned by the MEXT in Japan, Grant-in-Aid for Scientific Research (20650077) of the MEXT in Japan, and Grant for Industrial Technology Research (07C46050), New Energy and Industrial Technology Development Organization, Japan.

\section{References}

1. Peirs, J., Reynaerts, D., Van Brussel, H.: A Miniature Manipultor for Integration in a Selfpropelling Endoscope. Sensors and Actuators A 92, 343-349 (2001)

2. Nakamura, R., Kobayashi, E., Masamune, K., Sakuma, I., Dohi, T., Yahagi, N., Tsuji, T., Hashimoto, D., Shimada, M., Hashizume, M.: Multi-DOF forceps manipulator system for laparoscopic surgery. In: Delp, S.L., DiGoia, A.M., Jaramaz, B. (eds.) MICCAI 2000. LNCS, vol. 1935, pp. 653-660. Springer, Heidelberg (2000)

3. Yamashita, H., Hata, N., Kim, D., et al.: Handheld Laparoscopic Forceps Manipulator Using Multi-slider Linkage Mechanisms. In: Barillot, C., Haynor, D.R., Hellier, P. (eds.) MICCAI 2004. LNCS, vol. 3217, pp. 121-128. Springer, Heidelberg (2004)

4. Abbou, C.C., Hoznek, A., Salomon, L., et al.: Laparoscopic radical prostatectomy with a remote controlled robot. Journal of Urology 165, 1964-1966 (2001)

5. Ikuta, K., Sasaki, K., Yamamoto, K., et al.: Remote Microsurgery System for Deep and Narrow Space - Development of New Surgical Procedure and Microrobotic Tool. In: Dohi, T., Kikinis, R. (eds.) MICCAI 2002. LNCS, vol. 2488, pp. 163-172. Springer, Heidelberg (2002)

6. Nakamura, Y., Matsui, A., Saito, T.: Shape Memory-Alloy Active Forceps for Laparoscopic Surgery. In: Proc. 1995 IEEE International Conference on Robot and Automation, vol. 3, pp. 2320-2327 (1995)

7. Simaan, N., Taylor, R., Flint, P.: High Dexterity Snake-Like Robotic Slaves for Minimally Invasive Telesurgery of the Upper Airway. In: Barillot, C., Haynor, D.R., Hellier, P. (eds.) MICCAI 2004. LNCS, vol. 3217, pp. 17-24. Springer, Heidelberg (2004)

8. Sturges, R.H., Laowattana Jr., S.: A flexible, tendon-controlled device for endoscopy. In: Proc. 1991 IEEE International Conference on Robotics and Automation, vol. 3, pp. 2582-2591 (1991)

9. Amir, D., Howie, C., Alon, W., et al.: Percutaneous intrapericardial interventions using a highly articulated robotic probe. In: The First IEEE/RAS-EMBS International Conference on Biomedical Robotics and Biomechatronics, pp. 7-12 (2006)

10. Yagi, A., Matsumiya, K., Masamune, K., et al.: Rigid-Flexible Outer Sheath Model Using Slider Linkage Locking Mechanism and Air Pressure for Endoscopic Surgery. In: Larsen, R., Nielsen, M., Sporring, J. (eds.) MICCAI 2006. LNCS, vol. 4190, pp. 503-510. Springer, Heidelberg (2006)

11. Hata, N., Tokuda, J., Hurwitz, S., et al.: MRI-Compatible Manipulator with RemoteCenter-of-Motion Control. J. Magn. Reson. Imaging 27(5), 1130-1138 (2008)

12. De Visser, H., Heijnsdijk, E.A.M., Herder, J.L., et al.: Forces and displacements in colon surgery. Surgical Endoscopy 16, 1426-1430 (2002) 